\title{
On the global optimization of the beam angle optimization problem in intensity-modulated radiation therapy
}

\author{
H. Rocha ${ }^{1}$, J.M. Dias ${ }^{1,2}$, B. C. Ferreira ${ }^{3,4}$ and M. C. Lopes ${ }^{3,4}$ \\ ${ }^{1}$ INESCC, Coimbra, Portugal \\ ${ }^{2}$ FEUC, Coimbra, Portugal \\ 3 IPOC-FG, Coimbra, Portugal \\ ${ }^{4}$ I3N, Aveiro, Portugal
}

\begin{abstract}
The beam angle optimization (BAO) problem remains an important and challenging problem in intensitymodulated radiation therapy (IMRT) treatment planning. BAO consists on the selection of appropriate radiation incidence directions and may influence the quality of the IMRT plans, both to enhance organs sparing and to improve tumor coverage. This is a very difficult global optimization problem since it is a highly non-convex continuous optimization problem with many local minima. Many conventional BAO approaches are based on single-beam metrics to solve a relaxed combinatorial formulation of the BAO problem. Typically, the quality of the solutions obtained is not simply related to the final value of an objective function but rather judged by dose-volume histograms or considering a set of physical dose metrics. For that reason, and also due to the fact that the global optimum value is unknown, it is difficult to perceive, in medical physics point of view, how good a solution is or how much could it be improved. In a mathematical point of view, it is difficult to acknowledge how far a solution is from the global optimum. The objective of this paper is to present the difficulties in obtaining near global optimum solutions for the BAO problem, particularly when using single-beam approaches considering discrete subsets of all possible beam angles. The benefits of using a derivative-free approach for a continuous formulation of the BAO problem are discussed using a retrospective treated case of head-and-neck tumor at the Portuguese Institute of Oncology of Coimbra.
\end{abstract}

Keywords - Intensity-modulated Radiation Therapy, Beam Angle Optimization, Global Optimization, Single-beam Metrics, Derivative-free Optimization.

\section{INTRODUCTION}

The goal of radiation therapy is to deliver a dose of radiation to the tumor volume to sterilize all cancer cells minimizing the damages on the surrounding healthy organs and tissues. An important type of radiation therapy is intensitymodulated radiation therapy (IMRT), a modern technique where the radiation beam is modulated by a multileaf col- limator allowing the irradiation of the patient using nonuniform radiation fields from selected angles aiming to deliver a dose of radiation to the tumor minimizing the damages on the surrounding healthy organs and tissues. The IMRT treatment planning is usually a sequential process where initially a given number of beam directions are selected followed by the fluence map optimization (FMO) at those beam directions. Beam angle optimization (BAO) consists on the selection of appropriate radiation incidence directions and may influence the quality of the IMRT plans, both to enhance better organs sparing and to improve tumor coverage. The $\mathrm{BAO}$ problem is quite difficult since it is a highly non-convex optimization problem with many local minima - see Fig. 1. Regardless the evidence presented in the literature that appropriate radiation beam incidence directions can lead to a plan's quality improvement [1], in clinical practice, most of the time, the number of beam angles is assumed to be defined a priori by the treatment planner and the beam directions are still manually selected by the treatment planner in a timeconsuming trial and error iterative process.

In most of the previous works on beam angle optimization, the entire range $\left[0^{\circ}, 360^{\circ}\right]$ of gantry angles is discretized into equally spaced beam directions with a given angle increment, such as 5 or 10 degrees, where exhaustive searches are performed directly or guided by a variety of different heuristics including simulated annealing [2], genetic algorithms [3] or other heuristics incorporating a priori knowledge of the problem [4]. On the other hand, the use of single-beam metrics has been a popular approach to address the BAO problem as well, e.g., the concept of beam's-eye-view [5]. Despite the computational time efficiency of these approaches, the optimality of the solutions proposed cannot be guaranteed since the interplay between the selected beam directions is ignored.

It is well known that, when the BAO problem is not based on the optimal FMO solutions, the resulting beam angle set has no guarantee of optimality and has questionable reliability since it has been extensively reported that optimal beam angles for IMRT are often non-intuitive [6]. Therefore, the optimal FMO solutions will be used both to drive and com- 

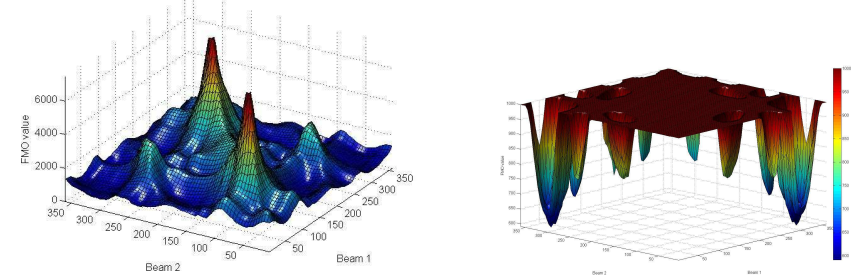

Fig. 1: 2-beam BAO surface (left) and truncated surface (right) to highlight the many local minima.

pare our BAO experiments. Typically, the quality of the solutions obtained is not simply related to the final value of an objective function but rather judged by dose-volume histograms or considering a set of physical dose metrics. For that reason, and also due to the fact that the global optimum value is unknown, it is difficult to perceive, in a medical physics point of view, how good a solution is or how much could it be improved. In a mathematical point of view, it is difficult to acknowledge how far a solution is from the global optimum.

The objective of this paper is to present the difficulties in obtaining near global optimum solutions for the BAO problem, particularly when using single-beam approaches considering discrete subsets of all possible beam angles. The benefits of using a derivative-free approach for a continuous formulation of the BAO problem are discussed using a retrospective treated case of head-and-neck tumor at the Portuguese Institute of Oncology of Coimbra. The paper is organized as follows. In the next section we describe the BAO problem and the FMO problem formulation used. Section 3 briefly presents the single-beam approaches tested and our derivative-free method proposed. Section 4 presents the obtained results. In the last section we have the conclusion.

\section{BeAm Angle Optimization Problem}

A quantitative measure is required to compare the quality of different sets of beam angles. For the reasons presented before, we will use the optimal solution of the FMO problem as measure of the quality of a given beam angle set. A convex penalty function voxel-based nonlinear model is used for the FMO problem [7]. In this model, each voxel is penalized according to the square difference of the amount of dose received by the voxel and the amount of dose desired/allowed for the voxel. This nonlinear formulation implies that a very small amount of underdose or overdose may be accepted in clinical decision making, but larger deviations from the desired/allowed doses are decreasingly tolerated.

The FMO optimal value is used to compare the solu- tions obtained by single-beam approaches and to drive the derivative-free approach we propose $[8,9,10]$. Our formulation of the BAO problem is briefly presented. Let us consider $n$ to be the fixed number of (coplanar) beam directions, i.e., $n$ beam angles are chosen on a circle around the computed tomography (CT)-slice of the body that contains the isocenter (usually the center of mass of the tumor). In our formulation we consider all continuous $\left[0^{\circ}, 360^{\circ}\right]$ gantry angles instead of a discretized sample. A basic formulation for the BAO problem is obtained by selecting an objective function such that the best set of beam angles is obtained for the function's minimum:

$$
\begin{array}{ll}
\min & f\left(\theta_{1}, \ldots, \theta_{n}\right) \\
\text { s.t. } & \theta_{1}, \ldots, \theta_{n} \in \mathbb{R}^{n} .
\end{array}
$$

Here, the objective $f\left(\theta_{1}, \ldots, \theta_{n}\right)$ that measures the quality of the set of beam directions $\theta_{1}, \ldots, \theta_{n}$ is the optimal value of the FMO problem for each fixed set of beam directions.

\section{BEAM ANGLE OPTIMIZATION APPROACHES}

\section{A. Single-beam approaches}

Two different single-beam approaches will be tested. One related to the beam's-eye-view concept and the other similar to the successful strategy used in [11].

The beam's-eye-view concept uses topographic criteria to rank the candidate beam directions. For IMRT, the geometrical considerations are not as important. Some variations of the beam's-eye-view concept consider dosimetric criteria to rank the candidate beam directions selecting those with higher scores [12]. Unlike conventional beams-eye-view (BEV) tools that considers only geometric criteria, beamseye-view dose metrics (BEVD) evaluate each possible beam direction using a score function that accounts for beam modulation. In IMRT, beam directions are often non-intuitive and have to go through sensitive organs to achieve an optimal compromise between target coverage and organs sparing, which makes the geometrical criteria used by BEV limited. An intensity-modulated beam can intercept a large volume of an organ at risk (OAR) or normal tissue and may not be necessarily a bad beam direction. The dose tolerances of the involved structures should be considered also when constructing a metric for measuring the quality of incident beam directions. Therefore, in IMRT, it is more appropriate to measure the quality of a radiation beam direction using a score function based on dosimetric criteria. A technique based on sensitive structures tolerance dose as a determinant factor for deliverable target dose $[5,12]$, denoted BEVD, was used to 
find a set of beams that are not too close to each other and have the largest scores. We should emphasize that a computationally intelligent algorithm should balance the BEVD scores and the beam interplay as a result of the overlap of radiation fields.

The other single-beam strategy tested is similar to the strategy used in [11] and can be described as follows:

1. Consider the 72 candidate beam angles $\{0,5, \ldots, 355\}$.

2. For $n=1$ beam direction:

Find the best beam irradiation direction, i.e., for the 72 beam angles in the candidate beam angle set, find the beam direction with lowest optimal FMO value.

3. For $n=2$ to $n=9$ beam directions:

Find the best set of $n$ directions considering fixed the $n-$ 1 beam directions determined in the previous iteration, i.e., find direction $n$ among the remaining $72-(n-1)$ beam directions similarly to procedure for $n=1$.

In the end of this sequential procedure, here denoted by SEQ, we will obtain "optimal" beam angle sets for $n=1$ to $n=$ 9 beam directions. This is a clear advantage of this strategy because it allows simultaneously an implicit optimization of the number of beam angles.

\section{B. Derivative-free approach}

Our derivative-free approach is based on a pattern search methods (PSM) framework. PSM are derivative-free optimization algorithms that require few function evaluations to progress and converge and have the ability to better avoid local entrapment making them a suitable approach for the resolution of the highly non-convex BAO problem $[8,9,10]$.

PSM are directional search methods that use positive bases to move in a direction that produces a decrease in the objective function. The main feature of a positive basis, that motivates PSM, is that for any given vector, in particular for the gradient vector, there is a vector of the positive basis that forms an acute angle with the gradient vector which means that it is a descent direction.

PSM are organized around two phases at every iteration: one that assures convergence to a local minimum (poll), and the other (search) where flexibility is conferred to the method allowing searches away from the neighborhood of the current iterate. Within the search step we use beams-eye-view dose metrics so that directions with larger dose metric scores are tested first improving results and computational time. This pattern search approach [8], denoted PSM-BEVD, was tested along with the single-beam strategies.

\section{Numerical Tests And Discussion}

A clinical example of a retrospective treated case of headand-neck tumor at the Portuguese Institute of Oncology of Coimbra is used to highlight the difficulties in obtaining near global optimum solutions for the BAO problem. The patients' CT set and delineated structures were exported via Dicom RT to a freeware computational environment for radiotherapy research. In general, the head-and-neck region is a complex area to treat with radiotherapy due to the large number of sensitive organs in this region (e.g. eyes, mandible, larynx, oral cavity, etc.). For simplicity, in this study, the OARs used for treatment optimization were limited to the spinal cord, the brainstem and the parotid glands. The tumor to be treated plus some safety margins is called planning target volume (PTV).

Our tests were performed on a $2.66 \mathrm{Ghz}$ Intel Core Duo PC with 3 GB RAM. In order to facilitate convenient access, visualization and analysis of patient treatment planning data, the computational tools developed within MATLAB and CERR [13] (computational environment for radiotherapy research) were used as the main software platform to embody our optimization research and provide the necessary dosimetry data to perform optimization in IMRT. The dose was computed using CERR's pencil beam algorithm (QIB). To address the convex nonlinear formulation of the FMO problem we used a trust-region-reflective algorithm (fmincon) of MATLAB 7.4.0 (R2007a) Optimization Toolbox. We choose to implement the incorporation of BEVD into the pattern search methods framework taking advantage of the availability of an existing pattern search methods framework implementation used successfully by us to tackle the BAO problem $[8,9,10]$ - the last version of SID-PSM $[14,15]$.

Typically, in head-and-neck cancer cases, patients are treated with 5, 7 or 9 equispaced beams in a coplanar arrangement. Therefore, results for the equispaced solution, denoted EQUI, for the BEVD solution, for the SEQ solution and for the PSM-BEVD solution are presented in Table 1 for 5, 7 and 9 beams. For this number of beams we can see that results obtained by PSM-BEVD are slightly better than SEQ solutions and way better than BEVD that struggles to be competitive with the traditional equispaced solutions. For larger numbers of beams the advantage of PSM-BEVD over SEQ is residual, indication that for larger number of beams the optimization process becomes harder. On the other hand, for single-beam approaches, particularly for SEQ, results tend to improve by increasing the number of beams. PSM-BEVD is also very competitive in terms of number of functions evaluations.

Solutions for 2 beams are also presented since the global optimum for the candidate beams $\{0,5, \ldots, 355\}$ was computed through exhaustive search (see Fig. 1) and we aim 
Table 1: BAO results for $n=2,5,7$ and 9 beams.

\begin{tabular}{|c|c|c|c|c|c|c|}
\hline \multirow[b]{2}{*}{$\mathrm{n}$} & \multirow{2}{*}{$\frac{\text { Equi }}{\text { Fval }}$} & \multirow{2}{*}{$\frac{\text { BEVD }}{\text { Fval }}$} & \multicolumn{2}{|c|}{ SEQ } & \multicolumn{2}{|c|}{ PSM-BEVD } \\
\hline & & & Fval & $\overline{\text { Fevals }}$ & Fval & Fevals \\
\hline 2 & 1278.6 & 936.5 & 624.5 & 143 & 588.1 & 84 \\
\hline 5 & 186.8 & 189.3 & 187.5 & 350 & 175.3 & 127 \\
\hline I & 173.5 & 174.8 & 168.1 & 483 & 162.4 & 158 \\
\hline 9 & 169.2 & 168.0 & 155.9 & 612 & 154.7 & 197 \\
\hline
\end{tabular}

to perceive how close solutions are to the global optimum. For 2 beams, the global optimum found in the candidate set $\{0,5, \ldots, 355\}$ was 591.4. Two interesting conclusions can be withdrawn: the PSM-BEVD solution is better than the global global optimum found in the candidate beam set and the single-beam approaches behave poorly for few beams. It is not straightforward to extrapolate conclusions for more beams but it is expected that differences between the solutions obtained and the global optimum increase since the optimization problem becomes harder with a larger search space to be explored.

\section{CONCLUSION}

The BAO problem is a continuous global highly nonconvex optimization problem known to be extremely challenging and yet to be solved satisfactorily. Many conventional BAO approaches are based on single-beam metrics to solve a relaxed combinatorial formulation of the $\mathrm{BAO}$ problem. We have shown, using an head-and-neck cancer case, that singlebeam strategies behave better for larger number of beams. On the other hand, PSM-BEVD has shown to yield solution of superior quality, in particular for smaller number of beams. This feature might be important for prostate or breast cancer cases where few beams are typically used. PSM-BEVD has shown ability to avoid local entrapment and efficiency by converging faster which is of the utmost importance in a busy clinical practice.

\section{ACKNOWLEDGEMENTS}

This work was supported by QREN under Mais Centro (CENTRO-07-0224-FEDER-002003) and FEDER funds through the COMPETE program and Portuguese funds through FCT under project grant PTDC/EIACCO/121450/2010. This work has also been partially supported by FCT under project grant PEst-C/EEI/UI0308/2011. The work of H. Rocha was supported by the European social fund and Portuguese funds from MCTES.

\section{REFERENCES}

1. Das SK, Marks LB. Selection of coplanar or non coplanar beams using three-dimensional optimization based on maximum beam separation and minimized nontarget irradiation Int J Radiat Oncol Biol Phys. 1997;38:643-655.

2. Bortfeld T, Schlegel W. Optimization of beam orientations in radiation therapy: some theoretical considerations Phys Med Biol. 1993;38:291304.

3. Dias JM, Rocha H, Ferreira BC and Lopes MC. A genetic algorithm with neural network fitness function evaluation for IMRT beam angle optimization Cent Eur J Oper Res. "in press"

4. D'Souza WD, Meyer RR, Shi L. Selection of beam orientations in intensity-modulated radiation therapy using single beam indices and integer programming Phys Med Biol. 2004;49:3465-3481.

5. Pugachev A, Xing L. Computer-assisted selection of coplanar beam orientations in intensity-modulated radiation therapy Phys Med Biol. 2001;46:2467-2476

6. Stein J, Mohan R, Wang XH, Bortfeld T, Wu Q, Preiser K, Ling CC, Schlegel W. Number and orientation of beams in intensity-modulated radiation treatments Med Phys. 1997;24:149-160.

7. Aleman DM, Kumar A, Ahuja RK, Romeijn HE, Dempsey JF. Neighborhood search approaches to beam orientation optimization in intensity modulated radiation therapy treatment planning $J$ Global Optim. 2008;42:587-607.

8. Rocha H, Dias JM, Ferreira BC and Lopes MC. Beam angle optimization for intensity-modulated radiation therapy using a guided pattern search method Phys Med Biol. 2013;58:2939-2953.

9. Rocha H, Dias JM, Ferreira BC, Lopes MC. Selection of intensity modulated radiation therapy treatment beam directions using radial basis functions within a pattern search methods framework $J$ Glob Optim. "in press"

10. Rocha H, Dias JM, Ferreira BC and Lopes MC. Pattern search methods framework for beam angle optimization in radiotherapy design Appl Math Comput. "in press"

11. Breedveld S, Storchi PRM, Keijzer M, Heemink AW, Heijmen BJM. A novel approach to multi-criteria inverse planning for IMRT Phys Med Biol. 2007;52:6339-6353.

12. Pugachev A, Xing L. Pseudo beam's-eye-view as applied to beam orientation selection in intensity-modulated radiation therapy Int J Radiat Oncol Biol Phys. 2001;51:1361-1370.

13. Deasy JO, Blanco AI, Clark VH. CERR: A Computational Environment for Radiotherapy Research Med Phys. 2003;30:979-985.

14. Custódio AL, Vicente LN. Using sampling and simplex derivatives in pattern search methods SIAM J Optim. 2007;18:537-555.

15. Custódio AL, Rocha H, Vicente LN. Incorporating minimum Frobenius norm models in direct search Comput Optim Appl. 2010;46:265-278.

Author: Humberto Rocha Institute: INESC-Coimbra Street: Rua Antero de Quental 199

City: Coimbra

Country: Portugal

Email: hrocha@mat.uc.pt 